\title{
El modelo Canvas como fundamento del plan de negocios para empresas de servicios de software de la ciudad de Cuenca
}

\section{The Canvas model as the foundation of the business plan for software services companies in the city of Cuenca}

\author{
Efraín Galo Cuzco Simbaña \\ ecuzcos@psg.ucacue.edu.ec \\ Universidad Católica de Cuenca \\ Ecuador \\ https://orcid.org/0000-0001-7664-3318 \\ Juan Carlos Erazo Álvarez \\ jcerazo@ucacue.edu.ec \\ Universidad Católica de Cuenca, Cuenca \\ Ecuador \\ https://orcid.org/0000-0001-6480-2270 \\ Cecilia Ivonne Narváez Zurita \\ inarvaez@ucacue.edu.ec \\ Universidad Católica de Cuenca, Cuenca \\ Ecuador \\ https://orcid.org/0000-0002-7437-9880 \\ Gonzalo Iván Andrade Mena \\ gonzaloandrade@ucacue.edu.ec \\ Universidad Católica de Cuenca, Cuenca \\ Ecuador \\ https://orcid.org/0000-0003-2777-4260
}

Recibido: 07 de septiembre de 2019

Aprobado: 28 de septiembre de 2019

\section{RESUMEN}

Los mecanismos que utilizan las empresas para generar sus ingresos es la base para el éxito, así como las herramientas para alcanzar los objetivos que se proponen de una manera más eficiente. El objetivo de la presente investigación es proponer un plan de negocios basándose en el modelo canvas para las empresas de servicios de software 
en la ciudad de Cuenca, que les permita generar los ingresos adecuados para permanecer en el mercado cada vez mas competitivo. La investigación fue de tipo mixta (cuali-cuantitativa), con una metodología de carácter descriptiva, para su análisis y diagnóstico de la situación se utilizó la técnica de la encuesta aplicada a 138 empresas que favoreció la identificación de las necesidades de éstas respecto al tipo de servicios que requieren de las organizaciones objeto de estudio de esta investigación. Los resultados más relevantes obtenidos tienen que ver con la necesidad de crear asociaciones, actividades y recursos claves, así como relacionarse con los clientes utilizando canales adecuados que permitan presentar una propuesta de valor.

Descriptores: Modelo; Herramienta; Empresa; Servicios; Canvas.

\begin{abstract}
The mechanisms that companies use to generate their income is the basis for success, as well as the tools to achieve the objectives that are proposed in a more efficient way. The objective of this research is to propose a business plan based on the canvas model for software services companies in the city of Cuenca, which allows them to generate adequate income to remain in the increasingly competitive market. The research was mixed (qualitative-quantitative), with a descriptive methodology, for its analysis and diagnosis of the situation, the survey technique applied to 138 companies was used, which favored the identification of their needs with respect to the type of services that require the organizations under study of this research. The most relevant results obtained have to do with the need to create associations, activities and key resources, as well as interact with customers using appropriate channels that allow presenting a value proposition
\end{abstract}

Descriptors: Model; Tool; Company; Services; Canvas.

\title{
INTRODUCCIÓN
}

El uso de la tecnología en las empresas se ha convertido en un factor clave y determinante para la generación de rentabilidad de las empresas, el uso de sistemas informáticos que funcionen las 24 horas, los 7 días de la semana, durante los 365 días del año; hoy en día, es crítico para las empresas.

Las empresas de servicios de software que sirven de apoyo a las empresas en la identificación de problemas en sus procesos críticos, que ayudan en la automatización de los procesos, haciéndolos más eficientes, a través de la implementación de software 
informático, que mejore la gestión de su inventario, sus recursos, sus clientes, además de potenciar sus productos y ampliar sus canales de ventas, ya sean estas pequeñas o medianas empresas (PYMES) es fundamental para su desarrollo.

La creciente tendencia hacia el comercio electrónico o e-commerce (electronic commerce en inglés) o conocidos también como negocios por Internet, negocios online, que consiste en la compra o venta de productos y/o servicios a través de medios electrónicos como lo es Internet, ofrece a las empresas la oportunidad de agrandar sus mercados y clientes, a través de canales digitales de ventas; la empresa de servicios de software provee a las empresas el sistema necesario para incluir sus productos $\mathrm{y} / \mathrm{o}$ servicios a través de Internet con la implementación de sitios web virtuales y/o aplicaciones para dispositivos móviles (Smartphone), donde las PYMES pueden ofertar sus productos, facilitando a los clientes la adquisición de estos, ofreciendo además ventajas como la posibilidad de seleccionar la formas de pago, diferentes opciones para la entrega de productos y/o pedidos, etc., Arraiz (2018).

La problemática identificada en la presente investigación es la baja participación en el mercado, lo que ocasiona que no se obtengan los ingresos necesarios para operar adecuadamente este tipo de empresas. Para resolver este problema se plantea la formulación de un plan de negocios mediante la utilización del modelo canvas.

\section{Los planes de negocios, generadores de ingresos para las empresas}

La creación de una empresa inicia con una idea, una idea que puede ser el resultado de un análisis del entorno en el que nos desenvolvemos, o simplemente de nuestra propia experiencia en la cual identificamos posibles necesidades que están pendientes por satisfacer y que muy posiblemente podrían ser explotadas comercialmente; esto no deja de ser una simple especulación mientras no se realice un estudio que respalde o definitivamente demuestre que lo que nos imaginamos al inicio como la mejor idea del mundo para ganar dinero en realidad no era tan buena idea como pensábamos, puesto 
que al ser ejecutada nos traería perdidas económicas así como también del tiempo invertido, en lugar de generar ganancias.

Incluso la idea más sencilla puede terminar generando pérdidas, por culpa de una idea mal planteada, o su vez poco analizada, las ideas de negocio debe ser realista y poder llevarse a la realidad, no es necesario encontrar un negocio que revolucione los mercados financieros o comerciales, sino de detectar un hueco, una oportunidad que nadie haya aprovechado (Zorita, 2016).

Las ideas de negocio pueden ser el resultado de nuestras fortalezas, capacidades, habilidades o experiencias; luego de la búsqueda de ideas de negocios y establecer la más idónea de acuerdo a nuestro perfil como emprendedores, es necesario realizar una planificación que encamine a hacer realidad esa idea, debemos planificar si queremos que nuestro negocio siga existiendo a lo largo de los años.

Planificar es definir lo que se debe hacer y asignar los recursos necesarios para hacerlo, la planificación traza el camino a seguir hacia la consecuencia del objetivo principal que es hacer realidad la idea negocio que se ha planteado. En el proceso de planificación se asigna los recursos y responsabilidades necesarios, la planificación es una función básica que permite que la empresa se prepare hoy para competir en escenarios futuros y pueda aprovechar las oportunidades, minimizando el riesgo inherente a las empresas, y teniendo planes de contingencia para hacer frente a los imprevistos que se presenten. Algunas consecuencias de la falta de planificación son:

Falta de control.

Muchas situaciones imprevistas

No existe medida para evaluar el éxito o fracaso de la gestión.

Falta de una guía de acción.

Peligrosa visión a corto plazo.

Falta de criterios para decidir inversiones y gastos.

Desperdicio de dinero, de tiempo y de oportunidades. 
De aquí la importancia de realizar un estudio previo a la implementación de una idea de negocio para conocer a detalle los aspectos que involucran la idea, a este estudio se le conoce como plan de negocios, que se define como "un documento que describe actividades para generar ganancias... a través de la satisfacción de necesidades mediante el ofrecimiento de bienes o servicios" (Moyano, 2015).

Un plan de negocios es un documento donde se describe las actividades comerciales a las que se dedicara la empresa, así como también los aspectos de producción, logísticos, de recursos humanos, financieros económicos, legales necesarios para la creación de la empresa, y que esta pueda generar ganancias.

Evans (2012) afirma que el "... plan de negocios debe acometer las siete C: un buen plan debe ser claro, cautivador, conciso, consistente, coherente y creíble. Pero por encima de todo, debe ser convincente, especialmente al evaluar el riesgo", ya que la razón de ser de un plan de negocios es convencer a un futuro inversionista, que el negocio que se le presenta es una oportunidad de generar ganancias; aunque como veremos más adelante en este mismo capítulo, un plan de negocios tiene diferentes objetivos, Chirinos (2016). Para poder llevar a cabo una actividad con éxito es necesario siempre la planificación y el apoyo de instrumentos que nos ayudan, nos orientan a clarificar las ideas para poder plantear objetivos o metas realistas, que nos ayude a estructurar las actividades, y en fin a organizar todos los factores que inciden en una idea de negocio o proyecto. Existen varias herramientas, metodologías o técnicas que nos pueden ayudar, sin embargo, en este documento se plantea el uso de los conocidos como planes de negocio y proyectos de inversión.

A continuación, se expone varias definiciones sobre un plan de negocio, que va desde conceptos, para que sirve, cual es el objetivo, para que se hace y cuál es el contenido fundamental que un plan de negocios debe tener, de igual manera luego se presenta una definición de proyectos de inversión para poder determinar cuál es la principal diferencia entre estos dos tipos de instrumentos. 
Existen varias definiciones de un plan de negocios autores que lo definen como un instrumento, como una herramienta, para determinar factibilidad de un negocio, para esto en un documento se redacta la planificación para llevar a cabo cierta actividad en cierto periodo en el futuro (Stutely, 2000).

Autores como Zorita (2016) estable a los planes de negocio también como planes de empresa donde se indica que "Es un documento dinámico, que se escribe y se reescribe, se modifica según se va consiguiendo información relevante para tomar decisiones sobre cada uno de los elementos y variables que los conforman." (p. 25)

Planear es una forma de anticipar el futuro, teniendo en cuenta esta consideración, plan de negocios es la planificación de una empresa o negocio en su conjunto, considerando cada una de sus partes esenciales como: Administración, Finanzas, Mercadotecnia, Operaciones, etc. (Goercke, Erazo, Narváez y Quevedo, 2019)

El plan de negocios es una herramienta que es utilizada para alcanzar los objetivos que se proponen de una manera más eficiente, a través de una adecuada planeación de la operación de cada una de las áreas de la empresa (Viniegra, 2011).

Un plan de negocios es la descripción del negocio que se piensa iniciar, que es una planificación de cómo se va a dirigir y desarrollarlo, así se tiene el conocimiento del futuro que le aguarda (Thomsen, 2009).

Un plan de negocio es un documento escrito de manera sencilla y precisa, y es una herramienta de gestión de la empresa que sirve de guía para el emprendedor o empresario implemente un negocio. Esta guía es un documento en el que se describe el negocio a realizar, analizando el mercado y se establecen las acciones que se realizarán a futuro, junto a las correspondientes estrategias para implementarlas. De esta manera, el plan de negocio, es también un instrumento de planificación que permite comunicar una idea de negocio para gestionar su financiamiento (Andía, 2012).

Los planes de negocio presentan diferentes definiciones que de fondo tienen un objetivo en común que es guiar a un empresario al éxito empresarial, evitar pérdidas o aumentar la rentabilidad de una empresa ya existente. 
Un plan de negocios es una herramienta que ayuda a las personas que buscan realizar algún emprendimiento, realizar un proceso de planeación que coadyuve a seleccionar el camino adecuado para conseguir las metas y objetivos, por lo que un plan de negocios se constituye en un medio para concretar ideas; una forma de poner estas ideas por escrito, en blanco y negro, de una manera formal y estructurada, por lo que se convierte en una guía de la actividad diaria del emprendedor, en resumen el plan de negocio es un documento que identifica, describe y analiza una oportunidad de negocio, examina la viabilidad técnica, económica y financiera de la misma, y desarrolla todos los procedimientos y estrategias necesarios para convertir la citada oportunidad de negocio en un proyecto empresarial concreto (Cosío, 2011)

La elaboración del plan de negocios define las etapas de desarrollo de un proyecto de una empresa y una guía que facilita la creación o el crecimiento de la misma, también es una carta de presentación para posibles inversionistas o para obtener financiación por medio de un préstamo; también minimiza la curva de aprendizaje, la incertidumbre y el riesgo del inicio de una empresa, también facilita el análisis de la viabilidad, factibilidad técnica y económica de un proyecto.

\section{Los modelos de negocios}

Al hablar de modelos de negocio podemos encontrar varias definiciones igual de importantes, sin embargo, podemos citar la definición de Osterwalder y Pigneur (2011) quienes establece que "un modelo de negocio describe las bases sobre las que una empresa crea, proporciona y capta valor" (p.14). Un concepto sencillo que a la vez simplifica el entendimiento de las bases sobre las cuales se puede crear una empresa. La forma de como las empresas hacen para generar sus ingresos es la base para el éxito de la empresa, por lo tanto tener claro este concepto ayuda a los gerentes a entender de mejor manera el funcionamiento del negocio, esto permite determinar las relaciones existentes entre los diferentes elementos que rodean la empresa, facilitando la creación 
de estrategias para la consecución de los objetivos empresariales, en definitiva el modelo de negocio es sin lugar a dudas un factor clave en la definición de una empresa.

El modelo de negocios es un tema que ha sido estudiado por varios autores, por citar algunos Porter (2016) propone el conocido modelo de negocio de las 5 fuerzas competitivas, donde establece que estas fuerzas son: la amenaza de productos sustitutos, la amenaza de nuevos competidores, la rivalidad de competidores, el poder de negociación de los proveedores y el poder de negociación de los clientes.

\section{Figura 1.}

Modelo de las Cinco Fuerzas Competitivas

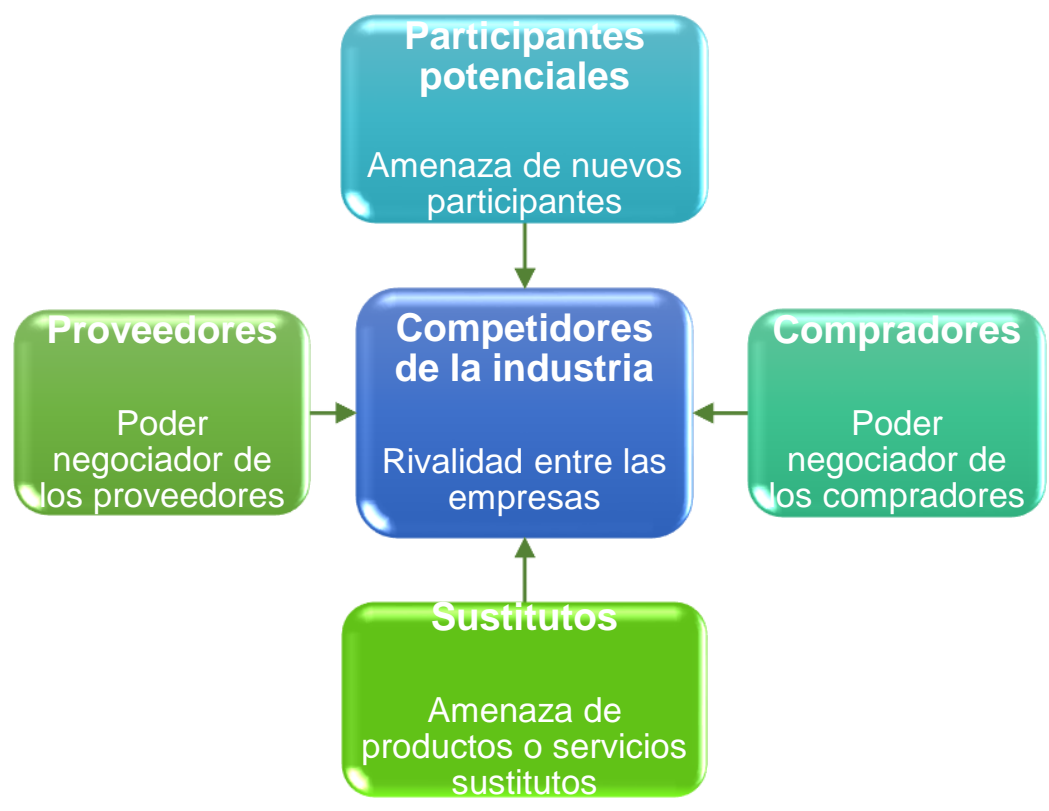

Fuente: Pietersma y Berg, 2016.

Otro modelo es el conocido como la estrategia del océano Azul, donde define la importancia de la innovación para abrir nuevos mercados, que permita evitar la competencia destructiva que existe en el mercado, esta estrategia propone cuatro acciones para generar una nueva propuesta de valor. Las acciones que se propone son: Eliminar algo que actualmente se está ofreciendo y que al eliminar supone un ahorro de costos, como se eliminó tenemos que crear una nueva propuesta que no se haya ofrecido 
hasta el momento. Reducir el impacto de ciertas variables que no favorecen actualmente a la propuesta de valor, en contraparte se debe incrementar las variables que ofrezcas valor a la propuesta (Kim y Mauborgne, 2005).

Al ejecutar las acciones correctamente supone la creación de una oferta de valor diferente donde la competencia existen es irrelevante porque en realidad no existe, al haber encontrado un nuevo mercado, que antes no existía. De esta manera podemos disminuir el riesgo que supone ingresar en mercados ya explotados donde la lucha por captar clientes es muy agresiva.

\section{Figura 2.}

El esquema de las cuatro acciones de la estrategia azul

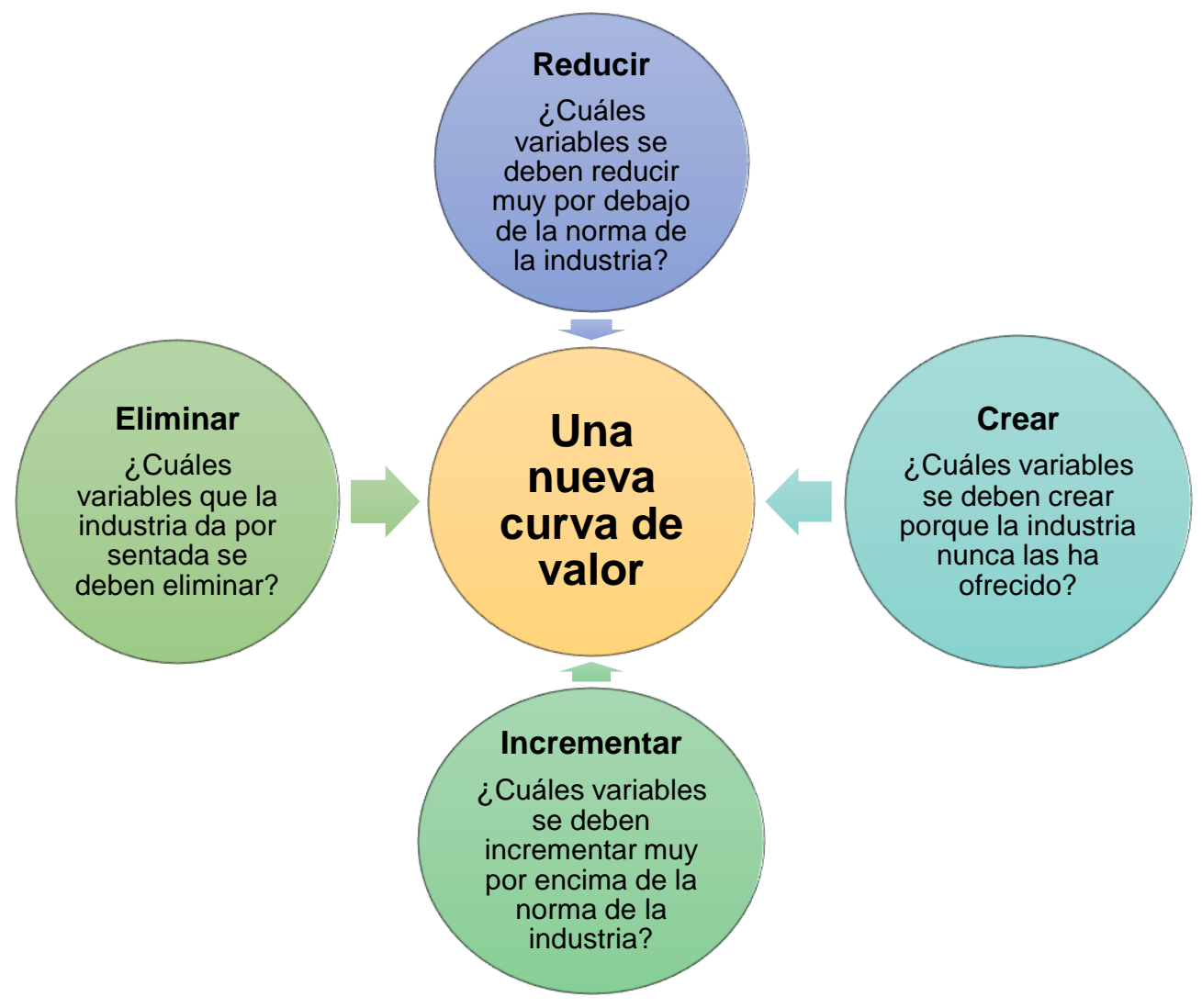

Otro modelo es el llamado "El método Lean Startup" donde Ries (2018) estable una metodología basada en un "aprendizaje validado", que consiste en ir validando poco a 
poco un producto, para esto podemos crear un prototipo del producto, darlo a conocer a los potenciales clientes, para que lo usen, lo prueben y nos den sus opiniones; esto nos permite ir verificando hipótesis sobre características o funcionalidades del producto para irlo adaptando a lo que el mercado demanda en ese momento.

La metodología Lean Startup propone 5 pasos fundamentales: La primera es plantearse una hipótesis a partir del problema a resolver explicar porque se pagaría por la oferta. Como segundo paso se debe validar la oferta creando el producto con las características mínimas requeridas, para conocer si el público acepta o no la oferta. El siguiente paso consiste en medir la hipótesis, identificando los KPI más adecuado para el producto y saber si se está cumpliendo con los objetivos. El cuarto paso consiste en generar un aprendizaje validado, esto es ir ajustando el producto y/o servicio para cada uno de los segmentos de clientes; por último, se regresa al primer paso, pero ya con un producto 0 servicio mejorado (Ries, 2018).

\section{Figura 3.}

Circuito de feedback de información crear-medir-aprender

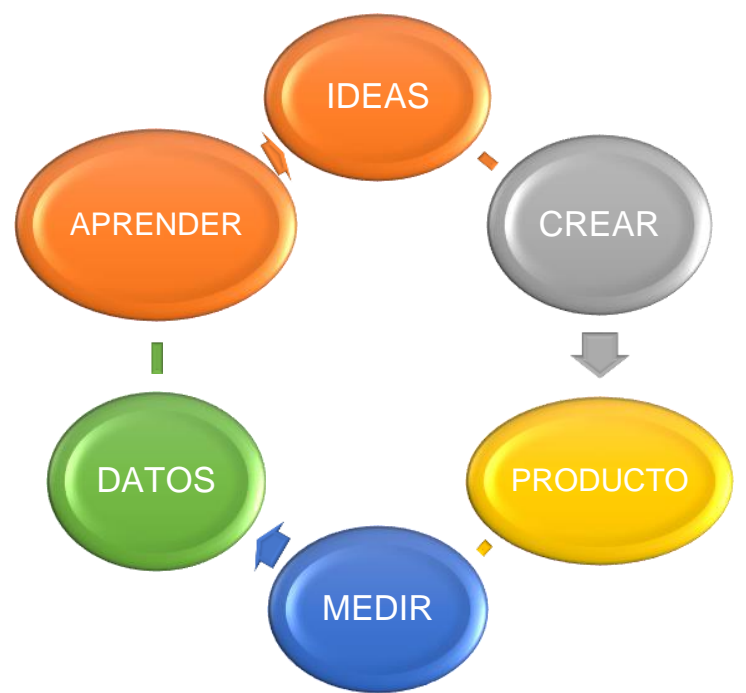

Luego de aplicar los pasos indicados en la metodología lean startup, y repetir el ciclo las veces que sean necesarias, obtenemos un mínimo producto viable, un producto con características y funcionalidades esenciales, que podemos lanzar al mercado para los 
potencias clientes, donde se recibe un feedback con el cual podemos mejorar el producto y lanzar una nueva versión, al realizar este proceso evitamos crear un producto únicamente con las percepciones propias de fabricantes o dueños de las empresas; y así evitamos que luego de un costoso proceso de fabricación, resulta que no era el producto que el cliente esperaba o necesitaba, teniendo en consecuencia que volver a replantear el producto.

Como se ha visto hasta el momento existen varios modelos o metodologías que sirven de apoyo a los empresarios, gerentes, emprendedores, etc., para la planificación estratégica de la empresa, sin embargo, a continuación, se describe el modelo canvas propuesto por Alexander Osterwalder que es una herramienta de gestión estratégica, que sirve de apoyo a quien empieza con la gran y emocionante aventura empresarial. En el 2010 Alexander Osterwalder, propone un lienzo para la descripción e innovación de modelos de negocio, la cual empieza a ser utilizada en diferentes empresas a nivel mundial, la aplicación exitosa del modelo en empresas muy reconocidas de todo el mundo ha hecho que este modelo canvas sea actualmente una de las principales herramientas estrategias que usan los gerentes para el análisis de la empresa; este lienzo es conocido como el modelo canvas.

El modelo canvas o llamado también metodología canvas, propone nueve bloques base para analizar el complejo funcionamiento de una empresa, estos bloques cubren los aspectos importantes que rodean a las empresas. Esta metodología permite describir, gestionar y trabajar con modelos de negocios, con el fin de desarrollar nuevas alternativas estratégicas. Osterwalder y Pigneur (2011) afirman "el modelo de negocio es una especie de anteproyecto de una estrategia que se aplicara en las estructuras, procesos y sistemas de una empresa" (p.15).

La metodología canvas es una herramienta simple de usar, ágil, versátil y muy útil para diseñar e innovar en los modelos de negocios empresariales de una forma visual. Permite tener un conocimiento claro de la relación entre factores como clientes, propuesta de valor, costos e ingresos, en un periodo de tiempo mucho más corto y a un 
menor coste en comparación a otras metodologías existentes. Todo lo anterior ha llevado a que hoy se esté convirtiendo en la herramienta de gestión estratégica preferida por las empresas (Dourado, 2015).

Entre las principales ventajas que presenta están la simplicidad de interpretación, gracias

a la distribución organizada de sus componentes. Presenta un enfoque integral y sistémico, haciendo más visible cualquier posible incoherencia entre sus componentes. Permite realizar cambios, probando diferentes alternativas y analizando sus posibles repercusiones. Permite un trabajo en equipo ya que facilita la generación e integración de ideas y aportes del grupo de trabajo. Este método es una poderosa herramienta para el análisis estratégico y empresarial: permitiendo realizar análisis FODA, análisis de mercado, analizar los competidores, clientes, proveedores, estructuras físicas y procesos (Cirugeda, 2015).

\section{Componentes de modelo canvas}

La metodología canvas se basa en el estudio de nueve componentes que conforman el lienzo canvas, para describir a detalle la estructura, los procesos, y los sistemas que conforma una empresa para su correcto funcionamiento; además permite conocer las relaciones existentes entre estos componentes de una manera clara y precisa. La siguiente figura define los componentes del modelo canvas para la generación de modelos de negocio.

\section{Figura 3.}

Modelo Canvas 


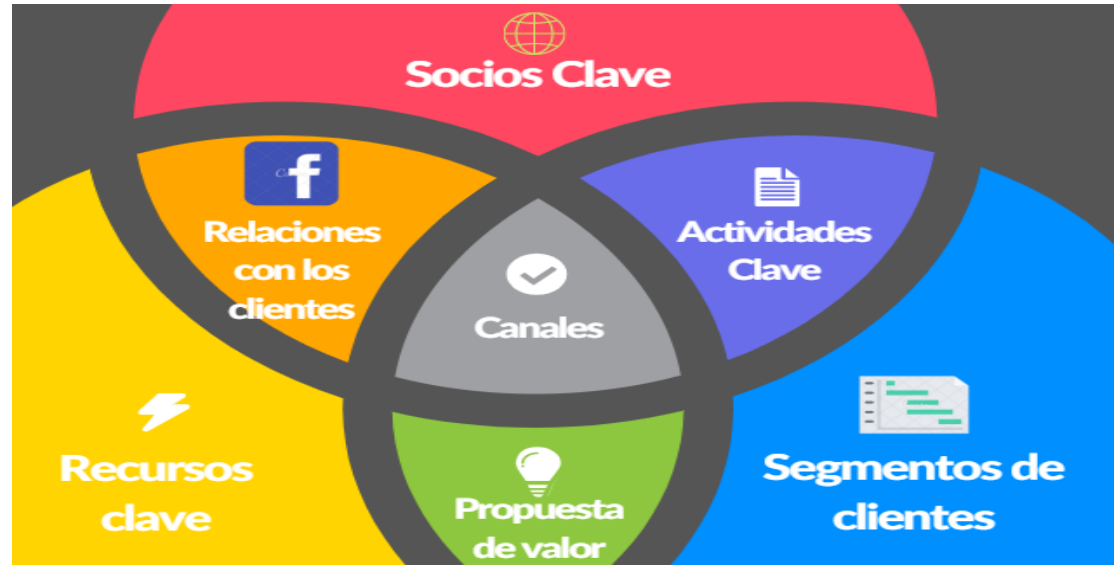

Esta metodología facilita la descripción y comprensión del modelo de negocio, por ende, facilita el trabajar con modelos de negocios de empresas nuevas o incluso ya existentes, ya que permite tener una vista integrada de todos los elementos que confirman la empresa, este modelo debe ser un proceso co-creación entre los diferentes niveles jerárquicos de la empresa, sin dejar de ser una herramienta intuitiva y visual que facilita el desarrollo de algo muy complejo, estos bloques cubren las cuatro áreas principales de una empresa: clientes, suministro, infraestructura y viabilidad financiera. La siguiente tabla muestra estos bloques de construcción agrupados por áreas de la empresa y el objetivo que persigue cada bloque (Dourado, 2015).

Tabla 1.

Las áreas de la empresa, los bloques del modelo canvas y objetivo

\begin{tabular}{|c|c|c|}
\hline Área & Bloques & Objetivo \\
\hline Oferta & Propuesta de valor & $\begin{array}{l}\text { Describir el conjunto de productos } \\
\text { y/o servicios que ofrece la empresa }\end{array}$ \\
\hline \multirow{3}{*}{ Clientes } & Canales & $\begin{array}{l}\text { Describir cómo la empresa se } \\
\text { comunica y se da a conocer a sus } \\
\text { segmentos de mercado }\end{array}$ \\
\hline & Segmentos de clientes & $\begin{array}{l}\text { Definir los grupos de personas a } \\
\text { los que la empresa pretende llegar }\end{array}$ \\
\hline & Relación con los clientes & $\begin{array}{l}\text { Presentar los tipos de relaciones } \\
\text { que una empresa establece con }\end{array}$ \\
\hline
\end{tabular}


CIENCIAMATRIA

Revista Interdisciplinaria de Humanidades, Educación, Ciencia y Tecnología

Año V. Vol. V. №1. Edición Especial. 2019

Hecho el depósito de ley: pp201602FA4721

ISSN-L: 2542-3029; ISSN: 2610-802X

Universidad Nacional Experimental Francisco de Miranda (UNEFM). Santa Ana de Coro. Venezuela

Efraín Galo Cuzco Simbaña; Juan Carlos Erazo Álvarez; Cecilia Ivonne Narváez Zurita; Gonzalo Iván Andrade Mena

\begin{tabular}{|c|c|c|}
\hline & & $\begin{array}{l}\text { cada uno de los segmentos de } \\
\text { mercado }\end{array}$ \\
\hline \multirow{3}{*}{ Infraestructura } & Socios Clave & $\begin{array}{c}\text { Establecer la red de proveedores y } \\
\text { socios estratégicos para hacer el } \\
\text { trabajo }\end{array}$ \\
\hline & Recursos Clave & $\begin{array}{c}\text { Resaltar los activos más } \\
\text { importantes para el funcionamiento } \\
\text { del negocio }\end{array}$ \\
\hline & Actividades Clave & $\begin{array}{c}\text { Describir las cosas más } \\
\text { importantes que una empresa debe } \\
\text { hacer }\end{array}$ \\
\hline \multirow{2}{*}{ Viabilidad Financiera } & Estructura de costes & $\begin{array}{c}\text { Cubrir todos los costes } \\
\text { involucrados en el funcionamiento } \\
\text { del negocio }\end{array}$ \\
\hline & Fuentes de Ingresos & $\begin{array}{l}\text { Analizar el beneficio económico } \\
\text { que una empresa obtiene a partir } \\
\text { de cada segmento de cliente }\end{array}$ \\
\hline
\end{tabular}

Osterwalder y Pigneur, (2011) crean un lienzo donde distribuye los nueve bloques de construcción de un modelo de negocio de manera simple y didactica, que facilita describe, analiza y dibuja los modelos de negocio, a continuación se presenta la figura del lienzo:

Figura 4.

Lienzo del modelo de negocio

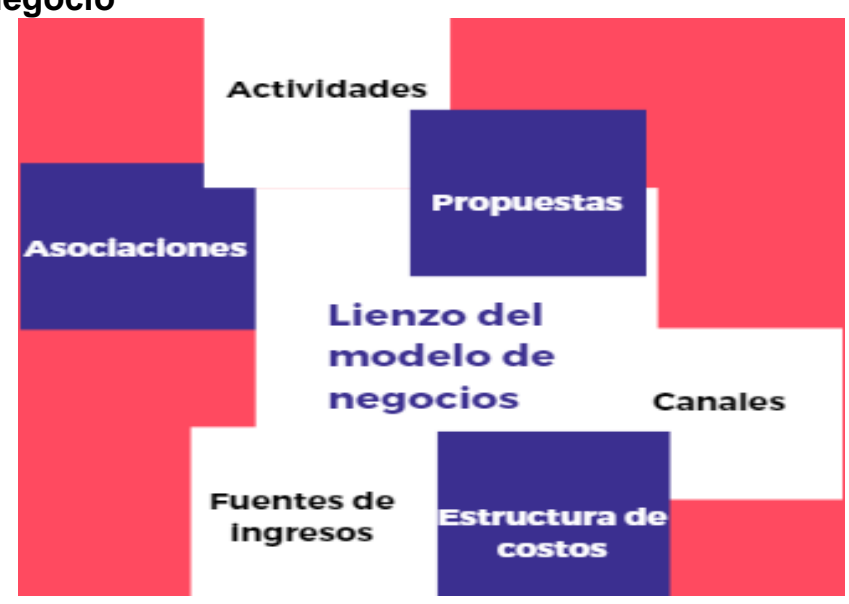

Fuente: Osterwalder y Pigneur, 2011. 


\section{METODOLOGÍA}

En este contexto la investigación que se realizó fue de tipo no experimental, ya que se realizó sin manipular deliberadamente alguna de las variables que influyen en la investigación. (Hernández Sampieri, Fernández y Baptista, 2015) afirma que son: “(...) estudios en los que no hacemos variar en forma intencional las variables independientes para ver su afecto (...) Lo que hacemos en la investigación no experimental es observar fenómenos tal como se da en su contexto natural para analizarlos" (p152).

Para el tipo de investigación según el enfoque, fue necesario recopilar datos de diferentes fuentes de información y de varios tipos de datos. Hernández Sampieri menciona "Los métodos mixtos representan un conjunto de procesos sistemáticos, empíricos y críticos de investigación e implican la recolección y el análisis de datos cuantitativos y cualitativos, para realizar inferencias (...) y lograr un mayor entendimiento del fenómeno bajo estudio" ( $p$ 534)., en este sentido fue necesario recopilar información y características de los elementos cuantitativos como los aspectos técnicos, financiero, de mercado; también se indago sobre cualidades deseadas acerca del servicio de publicidad digital, por lo tanto, se consideró la investigación con un enfoque mixto (Rodríguez, Erazo y Narváez, 2019).

La investigación según el alcance fue descriptivo-explicativo, los trabajos de investigación descriptivo-explicativo con los estudios descriptivos se busca especificar las propiedades, las características y los perfiles de personas, grupos, comunidades, procesos, objetos o cualquier otro fenómeno que se someta a un análisis" ( $p$ 92). mismo autor indica que los trabajos explicativos, van más allá de especificar las características o propiedades, están dirigidas a explicar por qué ocurre un fenómeno, a responder por las causas de los eventos, porqué se relacionan las variables. Por lo tanto, esto nos ayudó a conocer características fundamentales, rasgos y/o cualidades de la población objeto de estudio, los cual nos permitió determinar el mercado, el poder adquisitivo de las empresas, el perfil de los potenciales clientes de la agencia, la percepción de los empresarios acerca de los servicios deseados de la agencia de 
publicidad digital. Una vez especificado las diferentes características, se analizó las principales relaciones entre estas variables, explicando por qué sucedieron determinados fenómenos. La información recopilada se la realizo con técnicas propias de este tipo de investigación como lo son encuestas, la entrevista y la revisión documental necesaria.

La investigación según su finalidad, es considerada transversal, con un enfoque no experimental, como ya se indicó anteriormente, esto debido a que se analiza y describe las variables en un momento de tiempo específico único, es como "tomar una fotografía" de algo que sucede en un determinado tiempo (Sampieri, Fernández y Baptista, 2015). El plan de negocios está orientada a la creación de una agencia que ofrecerá servicios de publicidad digital para las empresas de la ciudad de cuenca, en este contexto es necesario establecer las principales características de las empresas que serán nuestra población.

Para este análisis se han considerado únicamente las empresas de la ciudad de Cuenca; que es el segmento de la población a la cual está dirigido los servicios de la nueva agencia. Para la muestra se ha tomado como base el registro de contribuyentes registrados en el servicio de rentas internas; filtrando esta base de empresas donde se considera únicamente las empresas en estado activas, y que actualmente estén con la condición de que no se encuentre suspendida. Con estas condiciones el total de empresas según el registro del SRI superan las 90,000 empresas, como se puede apreciar en la siguiente tabla agrupada por parroquias de la ciudad: 
CIENCIAMATRIA

Revista Interdisciplinaria de Humanidades, Educación, Ciencia y Tecnología

Año V. Vol. V. №1. Edición Especial. 2019

Hecho el depósito de ley: pp201602FA4721

ISSN-L: 2542-3029; ISSN: 2610-802X

Universidad Nacional Experimental Francisco de Miranda (UNEFM). Santa Ana de Coro. Venezuela

Efraín Galo Cuzco Simbaña; Juan Carlos Erazo Álvarez; Cecilia Ivonne Narváez Zurita; Gonzalo Iván Andrade Mena

Tabla 2.

Número de empresas en Cuenca por parroquia

\begin{tabular}{|c|c|}
\hline BAÑos $\quad$ Parroquia & Empresa \\
\hline $\begin{array}{l}\text { BAÑOS } \\
\text { BELLAVSTA }\end{array}$ & $\begin{array}{l}4181 \\
3875\end{array}$ \\
\hline CAÑARIBAMBA & 2210 \\
\hline $\mathrm{CHAUCHA}$ & 64 \\
\hline CHECA (JIDCAY) & 169 \\
\hline CHIQUINTAD & 437 \\
\hline CUENCA & 984 \\
\hline CUMBE & 475 \\
\hline E L BATAN & 5243 \\
\hline EL SAGR ARIO & 5561 \\
\hline EL VE CINO & 5900 \\
\hline GIL RAMIRE Z DAVALOS & 2434 \\
\hline HERMANO MIGUEL & 1861 \\
\hline HU AYNACAPAC & 5971 \\
\hline LLAC AO & 557 \\
\hline MACH ANGARA & 2133 \\
\hline MOLLETURO & 484 \\
\hline MONAY & 3393 \\
\hline NU LTI & 1101 \\
\hline OCT AVIO CORDERO PALACIOS & \\
\hline (SAN ROSA) & 143 \\
\hline PAC CHA & 556 \\
\hline QUIN GE O & 154 \\
\hline RIC AU RTE & 2983 \\
\hline SAN BLAS & 3774 \\
\hline SAN JOAQUIN & 1524 \\
\hline SAN SE BASTIAN & 7138 \\
\hline SANTA ANA & 391 \\
\hline SAYAUSI & 1938 \\
\hline SIDCAY & 490 \\
\hline SININCAY & 1598 \\
\hline SUCRE & 5712 \\
\hline TARQUI & 897 \\
\hline TOTORACOCHA & 5605 \\
\hline TURI & 1022 \\
\hline VALLE & 2229 \\
\hline VICTORIA DEL PORTETE & \\
\hline (IRQUIS) & 338 \\
\hline YANUNCAY & 8619 \\
\hline Total, General & 92144 \\
\hline
\end{tabular}

Fuente: Elaboración Propia basado en registro de empresas del SRI en la ciudad de Cuenca provincia del Azuay al año 2019.

El presente trabajo investigativo con enfoque cuantitativa es necesario analizar un grupo de datos de toda la población; para el cálculo de esta muestra se empleó la formula estadística para muestras finitas, ya que el tamaño de la población está determinado. Para el cálculo se aconseja un nivel de confianza del 95\%, con un error máximo admisible de $5 \%$. A continuación, se detalla la fórmula utilizada para el cálculo de la muestra: 
Donde $\mathrm{n}, \mathrm{z}, \mathrm{p}, \mathrm{q}, \mathrm{N}$ representan:

$$
n=\frac{z^{2} * p * q * N}{e^{2}(N-1)+z^{2} * p * q}
$$

$\mathrm{Z}=$ nivel de confianza elegido $95 \%$ (equivalente a $Z=1.96$ )

$\mathrm{p}=$ porcentaje de inasistencia $50 \%$ (equivalente a $\mathrm{p}=0.50$ )

$\mathrm{q}=$ porcentaje complementario $\mathrm{p}-\mathrm{q}=50 \%$ (equivalente $\mathrm{a} \mathrm{q}=0.50$ )

$\mathrm{N}=$ tamaño de la población $(\mathrm{N}=92144)$

e= error máximo permitido de $5 \%$ (equivalente a e=0.05)

$\mathrm{n}=$ tamaño de la muestra resultante

Los valores indicados son los recomendados, substituyendo los valores en la formula se tiene como resultado que el número de muestras es igual a 138, lo cual indica que para realizar esta investigación se tuvo que realizar 138 encuestas a las empresas de la ciudad de Cuenca, para determinar la demanda del servicio de publicidad digital y/o diseño web.

Una vez conocido el número de encuestas a realizar se ha decidido realizar un muestreo probabilístico aleatorio, ya que todas las empresas de la población tienen la misma probabilidad de ser seleccionada.

Para la aplicación de las 138 encuestas se realizó de manera personal, puesto que se cuenta con las direcciones físicas de las empresas; para seleccionar las empresas de cada parroquia se ha realizado un muestreo por conveniencia.

\section{RESULTADOS}

Respecto al tiempo de funcionamiento de los negocios de entre 5 y 10 años están el $68 \%$, respecto a la facturación mensual el $49 \%$ generan más de 10.000 dólares. Apenas el $28 \%$ utiliza la publicidad digital, los demás la tradicional. Sin embargo, el $85 \%$ considera que la de mayor impacto es la publicidad digital.

Respecto a la percepción de los costos por servicios de las empresas de software: 
CIENCIAMATRIA

Revista Interdisciplinaria de Humanidades, Educación, Ciencia y Tecnología

Año V. Vol. V. №1. Edición Especial. 2019

Hecho el depósito de ley: pp201602FA4721

ISSN-L: 2542-3029; ISSN: 2610-802X

Universidad Nacional Experimental Francisco de Miranda (UNEFM). Santa Ana de Coro. Venezuela

Efraín Galo Cuzco Simbaña; Juan Carlos Erazo Álvarez; Cecilia Ivonne Narváez Zurita; Gonzalo Iván Andrade Mena

\begin{tabular}{cc}
\hline Opciones de Respuesta & Respuestas \\
\hline Bajo & 25 \\
Normal & 33 \\
Alto & 42 \\
No sabe & 38 \\
\hline
\end{tabular}

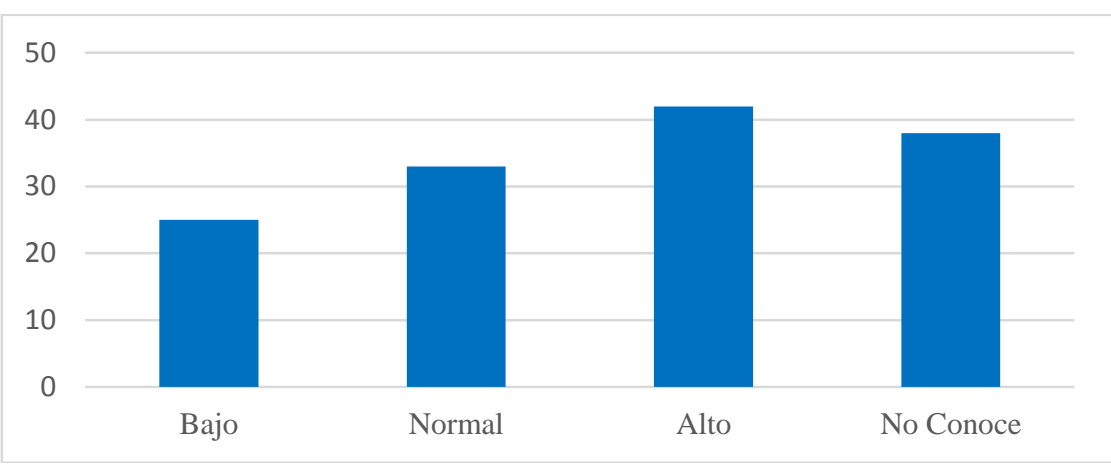

Fuente: Elaboración propia en base al resultado de las encuestas.

Según las respuestas obtenidas en las encuestas, se evidencia que la mayoría de las empresas asume que los costos por este tipo de servicios son altos.

En función a las necesidades que tienen las empresas para contratar los servicios de empresas de servicios de software:

\begin{tabular}{lc}
\hline \multicolumn{1}{c}{ Opciones de Respuesta } & Respuestas \\
\hline Incrementar ventas & 51 \\
Presencia en redes sociales & 21 \\
Tener una tienda online & 37 \\
Incrementar nuevos clientes & 29
\end{tabular}

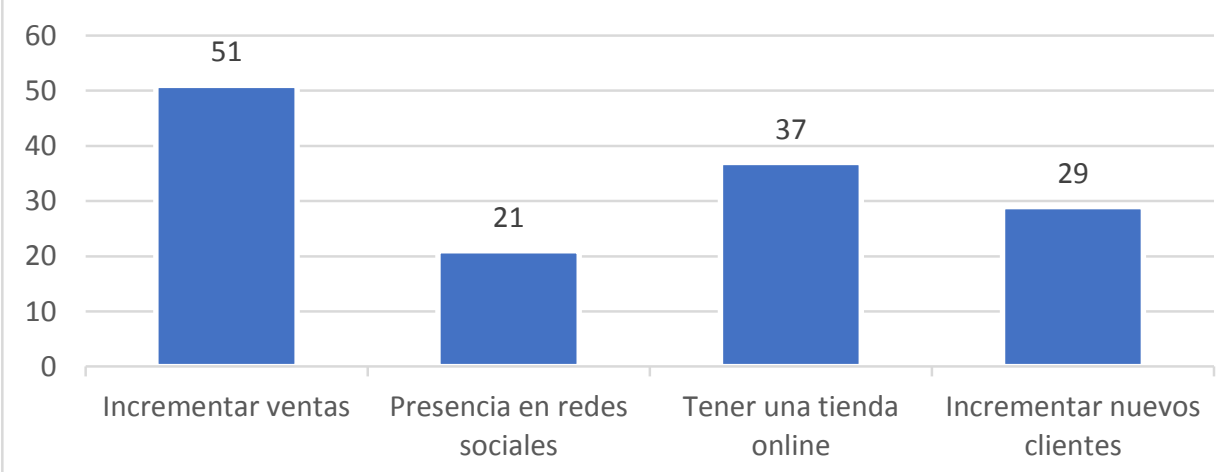

Fuente: Elaboración propia en base al resultado de las encuestas. 
CIENCIAMATRIA

Revista Interdisciplinaria de Humanidades, Educación, Ciencia y Tecnología

Año V. Vol. V. №1. Edición Especial. 2019

Hecho el depósito de ley: pp201602FA4721

ISSN-L: 2542-3029; ISSN: 2610-802X

Universidad Nacional Experimental Francisco de Miranda (UNEFM). Santa Ana de Coro. Venezuela

Efraín Galo Cuzco Simbaña; Juan Carlos Erazo Álvarez; Cecilia Ivonne Narváez Zurita; Gonzalo Iván Andrade Mena

La necesidad más importante que consideran las empresas es el incremento de ventas de los productos o servicios ofertados, es decir se contrata publicidad con el fin prioritario de incrementar las ventas.

De acuerdo a los resultados obtenidos, se confirma la necesidad de plantear un modelo de plan de negocios que permita generar ingresos y como consecuencia el aumento de la participación en el mercado. A continuación, se presenta una propuesta de plan de negocios mediante la utilización del modelo Canvas:

Figura 5.

Modelo Canvas propuesto a las empresas de servicios de software

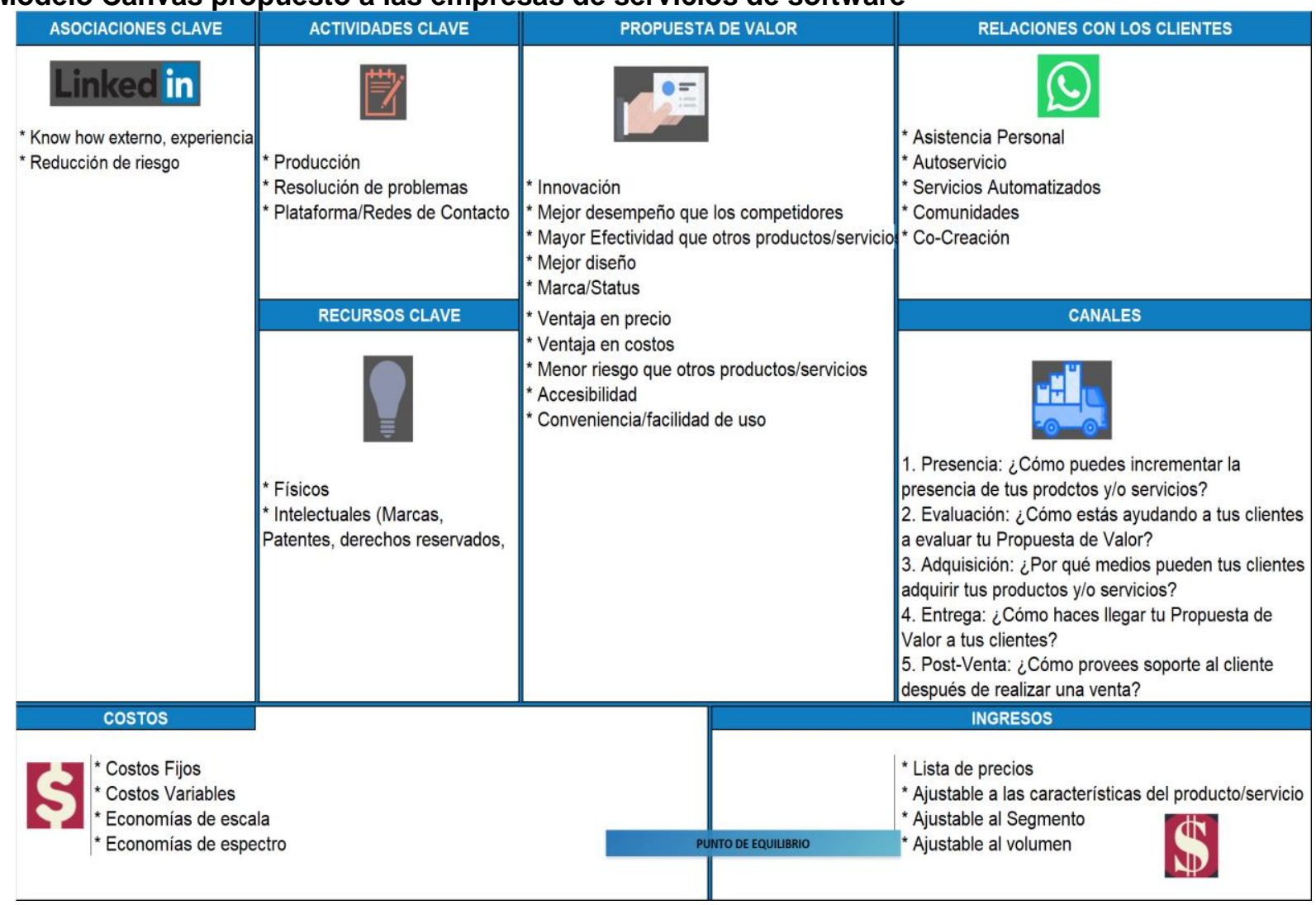

Fuente: MEP (2019). 


\section{CONCLUSIONES}

Las empresas en Cuenca muestran un claro interés acerca de la publicidad en medios digitales, no solo por el costo que esto representa con respecto a la publicidad tradicional, sino más bien debido al cambio tecnológico que se está experimentando en la actualidad, donde ahora es posible encontrar casi todo en internet, como en redes sociales, por lo tanto, las empresas que quieran dar a conocer sus productos y/o servicios deberán propiciar su presencia digital en el mercado. Es importante conocer las expectativas de las empresas acerca de la publicidad digital, para de esta manera las empresas puedan obtener el beneficio que ellas esperan conseguir al contratar un servicio de publicidad digital, donde por medio de una asesoría correcta de acuerdo al tipo de empresa, se alcance a cubrir con sus exigentes expectativas.

El modelo Canvas provee a cualquier tipo de empresa las herramientas necesarias para generar recursos que permitan satisfacer las necesidades de sus clientes, mediante el uso adecuado de los canales de distribución utilizando asociaciones que permitan elaborar propuestas que generen valor considerando los costos y los ingresos manteniendo el equilibrio financiero que se necesita para mantenerse en el mercado.

\section{REFERENCIAS CONSULTADAS}

1. Andía, V. W. (2012). Proyectos de inversión. Lima: El saber.

2. Arraiz, A. (2018). Framerwork de desarrollo de Proyectos Sociotecnológicos basado en la notación de Metamodelos de procesos de Ingeniería de Software (spem 2.0). Revista Arbitrada Interdisciplinaria Koinonía, 3(6), 79-95. Recuperado de

https://fundacionkoinonia.com.ve/ojs/index.php/revistakoinonia/article/view/141/1 $\underline{21}$

3. Cirugeda, E. (29 de 04 de 2015). Rankia: Blog Autónomos, emprendedores y empresas. Obtenido de Franquicias, Subvenciones, Negocios rentables, Startup: https://www.rankia.com/blog/autonomos-y-emprendedores/2766918-que$\underline{\text { modelo-canvas }}$ 
4. Cosio Hurtado, J. M. (2011). Los proyectos y los planes de negocio. Cochabamba: Universidad Católica Boliviana San Pablo.

5. Chirinos, A. (2016). La innovación como factor clave de éxito en la gerencia de las empresas de producción social venezolana. CIENCIAMATRIA, 2(2), 97-106. https://doi.org/10.35381/cm.v2i2.55

6. Dourado, D. (2015). Los modelos de negocios de las agencias creativas digitales de Brazil y España: UN estudio exploratorio basado en la metodología Business Model Canvas. Getafe: Universidad Carlos III de Madrid.

7. Evans, V. (2012). El plan de negocio. Madrid: Pearson.

8. Goercke Villarreal, J. R., Erazo Álvarez, J. C., Narváez Zurita, C. I., \& Quevedo Vázquez, J. O. (2019). Plan de negocio, proyecto constructora BellaTerra en la ciudad de Cuenca, Ecuador. Visionario Digital, 185.

9. Hernández Sampieri, Fernández Collado, \& Baptista. (2015). Metodología de la investigación. México: MacGraw Hill.

10. Kim, W., \& Mauborgne, R. (2005). La estrategia del océano azul. MA: Harvard Bussines School Press.

11. Moyano Castillejo, L. E. (2015). Plan De Negocios. Lima: Macro EIRL.

12. Osterwalder, A., \& Pigneur, Y. (2011). Generación de modelos de negocio. Barcelona: Centro Libros PAPF, S.L.U.

13. Pietersma, P., \& Berg, G. (2016). Modelos Clave de negocios: Más de 75 modelos que todo gerente necesita saber. Ciudad de México: Trillas.

14. Porter, M. E. (2016). Ser competitivo. Barcelona: Deusto.

15. Propia, M. E. (2019). Mi empresa propia. Obtenido de https://mep.pe/

16. Ries, E. (2018). El método LEAN STARTUP. Cómo crear empresas de éxito utilizando la innovación continua. Lima: Planeta Perú S.A.

17. Rodríguez, D., Erazo, J., \& Narváez, C. (2019). Técnicas cuantitativas de investigación de mercados aplicadas al consumo de carne en la generación millennial de la ciudad de Cuenca (Ecuador). Espacios, 20. 
18. Stutely, R. (2000). Plan de negocios, la estrategia inteligente. México: Pearson.

19. Thomsen, M. (2009). El plan de negocios dinámico. Dinamarca: dynamicbusinessplan.

20. Viniegra, S. (2011). Entendiendo el Plan de Negocios. Malaga: Lulu.com.

21.Zorita Lloreda, E. (2016). Plan de negocios. Colombia: ESIC Editorial.

\section{REFERENCES CONSULTED}

1. Andía, V. W. (2012). Investment projects. Lima: The knowledge.

2. Arraiz, A. (2018). Framerwork of development of Sociotechnological Projects based on the notation of Metamodels of Software Engineering processes (spem 2.0). Interdisciplinary Arbitrated Review Koinonía, 3 (6), 79-95. Recovered from https://fundacionkoinonia.com.ve/ojs/index.php/revistakoinonia/article/view/141/1 $\underline{21}$

3. Cirugeda, E. (29 of 04 of 2015). Rankia: Autonomous blog, entrepreneurs and companies. Obtained from Franchises, Subsidies, Profitable Businesses, Startup: https://www.rankia.com/blog/autonomos-y-emprendedores/2766918-quemodelo-canvas

4. Cosio Hurtado, J. M. (2011). Projects and business plans. Cochabamba: San Pablo Bolivian Catholic University.

5. Chirinos, A. (2016). Innovation as a key success factor in the management of Venezuelan social production companies. SCIENCE, 2 (2), 97-106. https://doi.org/10.35381/cm.v2i2.55

6. Dourado, D. (2015). The business models of digital creative agencies in Brazil and Spain: AN exploratory study based on the Business Model Canvas methodology. Getafe: Carlos III University of Madrid.

7. Evans, V. (2012). The business plan Madrid: Pearson.

8. Goercke Villarreal, J. R., Erazo Álvarez, J. C., Narváez Zurita, C. I., \& Quevedo Vázquez, J. O. (2019). Business plan, BellaTerra construction project in the city of Cuenca, Ecuador. Digital Visionary, 185. 
9. Hernández Sampieri, Fernández Collado, \& Baptista. (2015). Investigation methodology. Mexico: MacGraw Hill.

10.Kim, W., \& Mauborgne, R. (2005). The blue Ocean Strategy. MA: Harvard Bussines School Press.

11. Moyano Castillejo, L. E. (2015). Business plan. Lima: Macro EIRL.

12. Osterwalder, A., \& Pigneur, Y. (2011). Generation of business models. Barcelona: Centro Libros PAPF, S.L.U.

13. Pietersma, P., \& Berg, G. (2016). Key Business Models: More than 75 models that every manager needs to know. Mexico City: Trillas.

14. Porter, M. E. (2016). To be competitive. Barcelona: Deusto.

15. Own, M. E. (2019). My own company. Obtained from https://mep.pe/

16. Ries, E. (2018). The LEAN STARTUP method. How to create successful companies using continuous innovation. Lima: Planeta Perú S.A.

17. Rodríguez, D., Erazo, J., \& Narváez, C. (2019). Quantitative techniques of market research applied to meat consumption in the millennial generation of the city of Cuenca (Ecuador). Spaces, 20.

18. Stutely, R. (2000). Business plan, smart strategy. Mexico: Pearson.

19. Thomsen, M. (2009). The dynamic business plan. Denmark: dynamicbusinessplan.

20. Viniegra, S. (2011). Understanding the Business Plan. Malaga: Lulu.com.

21.Zorita Lloreda, E. (2016). Business plan. Colombia: ESIC Editorial.

(C2019 por los autores. Este artículo es de acceso abierto y distribuido según los términos y condiciones de la licencia Creative Commons Atribución-NoComercial-Compartirlgual 4.0 Internacional (CC BY-NC-SA 4.0) (https://creativecommons.org/licenses/bync-sa/4.0/). 